\title{
A Monotonic Code for Sound Azimuth in Primate Inferior Colliculus
}

\author{
Jennifer M. Groh, Kristin A. Kelly, and Abigail M. Underhill
}

\begin{abstract}
We investigated the format of the code for sound location in the inferior colliculi of three awake monkeys (Macaca mulatta). We found that roughly half of our sample of 99 neurons was sensitive to the free-field locations of broadband noise presented in the frontal hemisphere. Such neurons nearly always responded monotonically as a function of sound azimuth, with stronger responses for more contralateral sound locations. Few, if any, neurons had circumscribed receptive fields. Spatial sensitivity was broad: the proportion of the total sample of neurons responding to a sound at a
\end{abstract}

\section{INTRODUCTION}

The ability to localize a sound in space is one of the most essential functions of our auditory systems. Our brains are proficient at inferring the direction of a sound based on its spectral content, as filtered by the pinnae and head, and the relative arrival time and pressure level at the two ears. Considerable progress has been made in identifying the brain areas involved in this process in birds and a variety of mammalian species (for reviews, see Brainard, 1994; Clarey, Barone, \& Imig, 1992; Irvine, 1992; Takahashi, 1989; Phillips \& Brugge, 1985; Masterton \& Imig, 1984). However, surprisingly little is known about the neural basis of sound localization in primates (for reviews, see Kelly, Metzger, Mullette-Gilman, Werner-Reiss, \& Groh, 2002).

In this study, we explored how neurons in the inferior colliculus (IC) of rhesus monkeys respond as a function of sound location. The IC is a nearly obligatory stopping point for auditory information ascending from the brainstem to auditory thalamus and cortex (for a review, see Masterton, 1992). As such, the IC is likely to play a role in either computing or relaying information concerning sound location to higher brain structures. Both lesion studies (Zrull \& Coleman, 1997; Kelly \& Kavanagh, 1994; Wagner, 1993; Jenkins \& Masterton, 1982) and singleunit recording studies in a variety of species (see Discussion) support this basic view, but little evidence concerning spatial sensitivity in the IC of the primate

Dartmouth College given location ranged from $30 \%$ for ipsilateral locations to $80 \%$ for contralateral locations. These findings suggest that sound azimuth is represented via a population rate code of very broadly responsive neurons in primate inferior colliculi. This representation differs in format from the place code used for encoding the locations of visual and tactile stimuli and poses problems for the eventual convergence of auditory and visual or somatosensory signals. Accordingly, models for converting this representation into a place code are discussed. exists (Groh, Trause, Underhill, Clark, \& Inati, 2001; Jay \& Sparks, 1987).

We were particularly interested in the coding format employed by primate IC neurons to represent sound location. We considered two broad classes of representational format: place (also called labeled line and vector) codes and rate (or scalar) codes. As many different classification schemes have been employed previously (for discussion, see Groh, 2001; Churchland \& Sejnowski, 1992), a precise definition of the terminology we use is in order.

A place code for sound location would consist of a population of neurons, each of which would respond most vigorously to a best sound location (or contiguous range of sound locations) and more weakly for sounds outside this range. The set of preferred sound locations or receptive fields of the entire population should be distributed across the range of possible sound locations in the environment, as illustrated in Figure 1A. (In visual, somatosensory, and oculomotor brain regions, such receptive fields are often topographically organized, but such topography is not a computational necessity of this coding format and will not be investigated here.)

A rate code differs from a place code in that the best sound location for the bulk of the population of neurons would be the same: the acoustic axis of the contralateral ear, where binaural difference cues, such as interaural level (ILD) or interaural timing (ITD) differences, reach their maximal values (Figure 1B). Responses would increase monotonically as a function of how close the sound source is to the contralateral ear. 


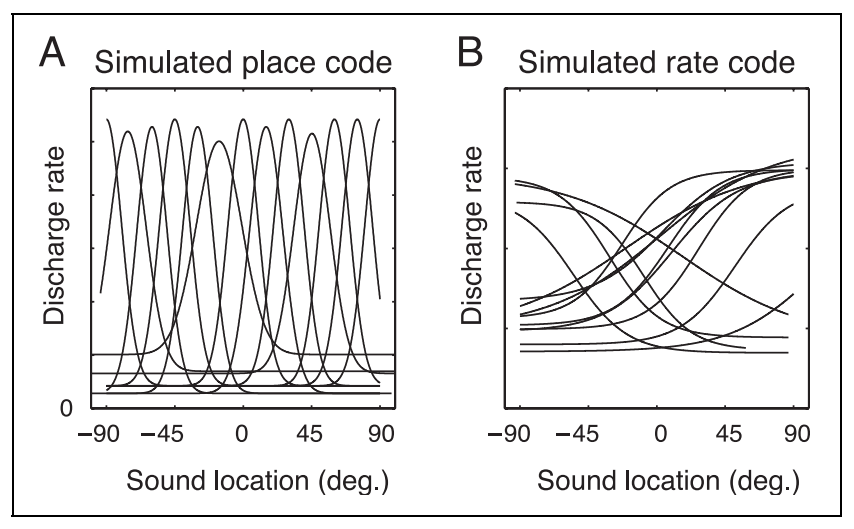

Figure 1. Illustration of the predicted responses of a place code (A) or a rate code (B) for sound location. (A) A place code would consist of an array of neurons with nonmonotonic sensitivity to sound location. Best sound locations would be distributed somewhat evenly across the frontal hemisphere (or potentially limited to the side of the frontal hemisphere contralateral to the recording site). (B). A rate code would consist of an array of neurons that respond monotonically as a function of sound azimuth in the frontal hemisphere. Best locations would likely cluster at the contralateral pole and possibly the ipsilateral pole as well.

Evidence for auditory place codes has been elusive in mammals. Currently, the most compelling example of a map of units with circumscribed receptive fields in the mammalian brain occurs in an oculomotor structure, the superior colliculus (Sterbing, Hartung, \& Hoffmann, 2002; Gaese \& Johnen, 2000; Meredith \& Stein, 1986; Middlebrooks \& Knudsen, 1984; King \& Palmer, 1983; King \& Hutchings, 1987; Wise \& Irvine, 1983, 1985). In mammalian IC, most studies investigating responses of neurons as a function of sound location or binaural difference cues report evidence consistent with both rate and place coding, but the evidence for rate coding is more prevalent. Free-field studies have noted that although circumscribed receptive fields appear to exist in some neurons, many others respond monotonically as a function of sound azimuth (Ingham, Hart, \& McAlpine, 2001; Delgutte, Joris, Litovsky, \& Yin, 1999; Aitkin, Gates, \& Phillips, 1984; Aitkin, Pettigrew, Calford, Phillips, \& Wise, 1985; Aitkin \& Martin, 1987; Moore, Hutchings, Addison, Semple, \& Aitkin, 1984; Bock \& Webster, 1974). Furthermore, the circumscribed receptive fields that do exist did not necessarily span a range of locations, but instead tended to center on the acoustical axis of the contralateral ear (Moore, Hutchings, et al., 1984; Moore, Semple, Addison, \& Aitkin, 1984; Semple, Aitkin, Calford, Pettigrew, \& Phillips, 1983), rendering their discharge patterns potentially more consistent with a rate code for azimuth in the frontal (and/or posterior) hemisphere. Only in the external nucleus of guinea pig IC (ICX) do receptive fields that span a range of space predominate (Binns, Grant, Withington, \& Keating, 1992) in a fashion that resembles the barn owl ICX (Knudsen \& Konishi, 1978).
A preliminary version of this work has appeared previously (Groh \& Underhill, 2001).

\section{RESULTS}

Sound source location was clearly an important factor governing the activity of primate IC neurons. Figure 2 shows a raster plot (A) and peristimulus time histogram (PSTH) (B) illustrating the dependence on sound
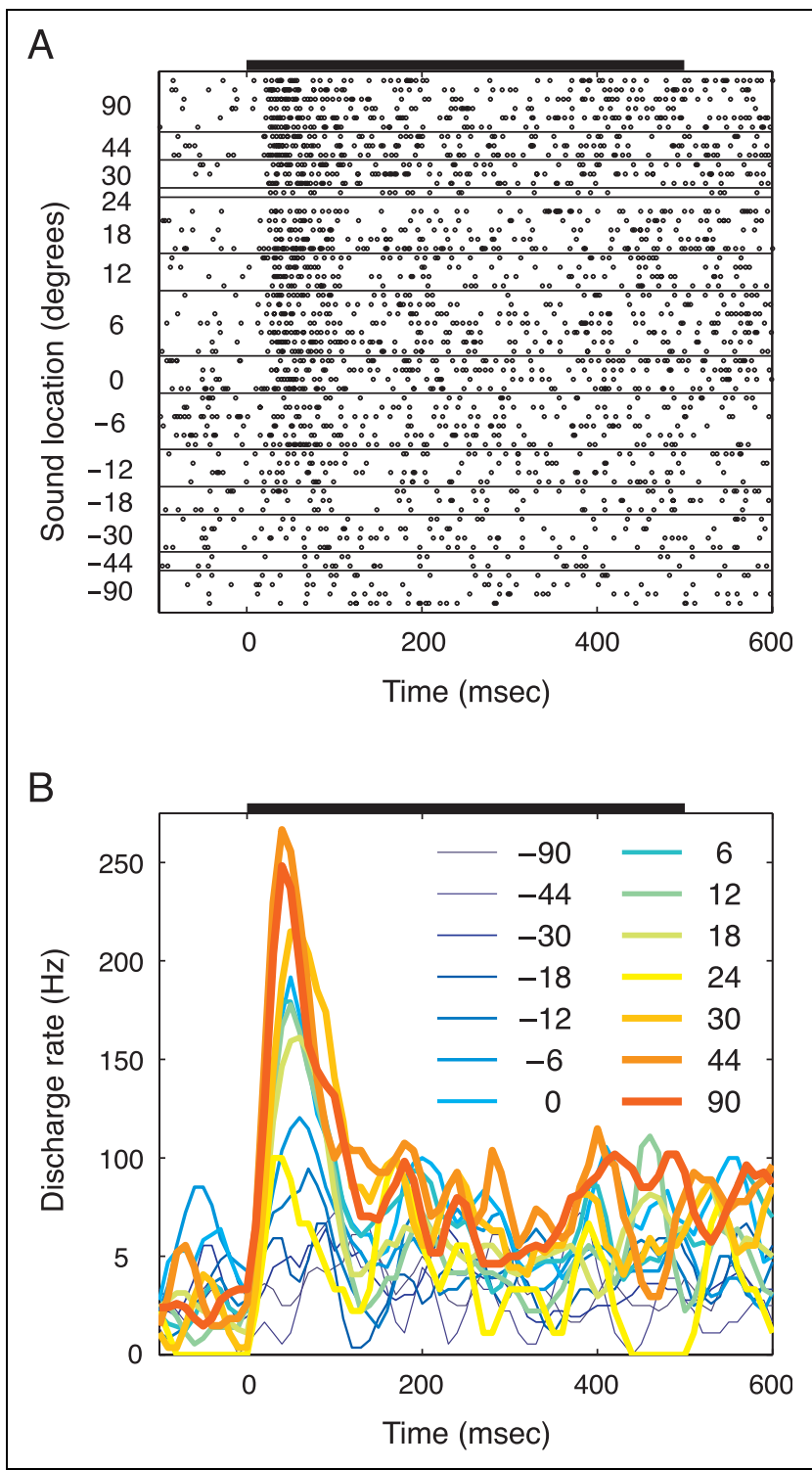

Figure 2. Example of an individual IC neuron with monotonic spatial sensitivity. (A) Raster plot by sound location, with positive numbers indicating contralateral locations. The dark horizontal bar indicates the duration of the stimulus. Each line of the raster plot is synchronized on the onset of a particular stimulus. (B) PSTH. This histogram was first constructed with 10-msec bins and then smoothed by convolution with a filter with values of $[1 / 9,2 / 9,1 / 3,2 / 9,1 / 9]$; thus, each trace represents the weighted average of the discharge rate during a sliding $50-\mathrm{msec}$ time window centered on the corresponding time value on the $\mathrm{x}$ axis. This accounts for the apparent rise in the discharge rates in the PSTH slightly before those in the rasters. 
location of the activity of one neuron. This neuron responded more vigorously for more contralateral sound locations than for ipsilateral sound locations. The effects of sound location were verified across the population of IC neurons using an analysis of variance (ANOVA), a conservative statistical approach that makes no assumptions regarding the shape of spatial sensitivity because sound location is treated as a categorical variable. According to the ANOVA, 49 of 99 neurons $(49 \%)$ showed a significant effect of sound location $(p<.05$, one-way ANOVA of effect of sound location on neural responses).

\section{Shape of Spatial Sensitivity}

As mentioned in the Introduction, neurons that form a place code for sound location must be tuned for locations within the frontal hemifield. Such neurons should therefore exhibit nonmonotonic responses as a function of sound azimuth. In contrast, neurons that form a rate code for sound location will respond monotonically as a function of sound azimuth. One can therefore obtain quantitative evidence concerning the rate and place coding hypotheses by comparing the adequacy of monotonic and nonmonotonic curve fits at capturing the relationship between discharge rate and azimuthal sound location. ${ }^{1}$

If neurons respond nonmonotonically as a function of sound location, they will generally require a nonmonotonic function to fit the relationship between discharge rate and sound location; a monotonic (e.g., sigmoidal or linear) function will be quite inadequate, as shown by the simulation in Figure 3A. In contrast, if neurons

Figure 3. Illustration of the use of curve fitting to distinguish between nonmonotonic and monotonic azimuthal response functions. (A) Simulation of an individual neuron with a nonmonotonic azimuthal response function. Variability was added to the simulated responses on individual trials by random draws from a normal distribution. Only the Gaussian function (thin line) adequately fit this response pattern; the sigmoidal function (thick line) was not successful. Gaussian-only fits can thus serve as evidence of a nonmonotonic code. (B) Simulation of an individual neuron with a sigmoidal-shaped azimuthal response function. Variability was added in the same manner as for the simulated neuron in (A). Sigmoidal (thick line) and Gaussian (thin line) functions fit the response pattern about equally well, and the shapes of these functions were very similar to one another. Sigmoidal and Gaussian fits that are equally good can thus serve as evidence of monotonicity. (C) Average discharge rate (minus baseline) versus sound location of the neuron shown in Figure 2 during the first 100 msec after stimulus onset. The response window was chosen based on inspection of an unsmoothed PSTH of all trials for this neuron. The fit of the sigmoidal (thick line) and Gaussian (thin line) functions are about equally good (note the $R^{2}$ values). As noted in the Methods, the baseline activity was determined for each trial prior to the onset of the first sound, a period that is not necessarily visible on the raster plot in Figure 2A, which is synchronized on each sound presentation. The number of data points appears to differ from the number of lines in the raster in Figure 2A because several identical data points are superimposed on one another in this graph.
A

\section{Simulated non-monotonic neuron}

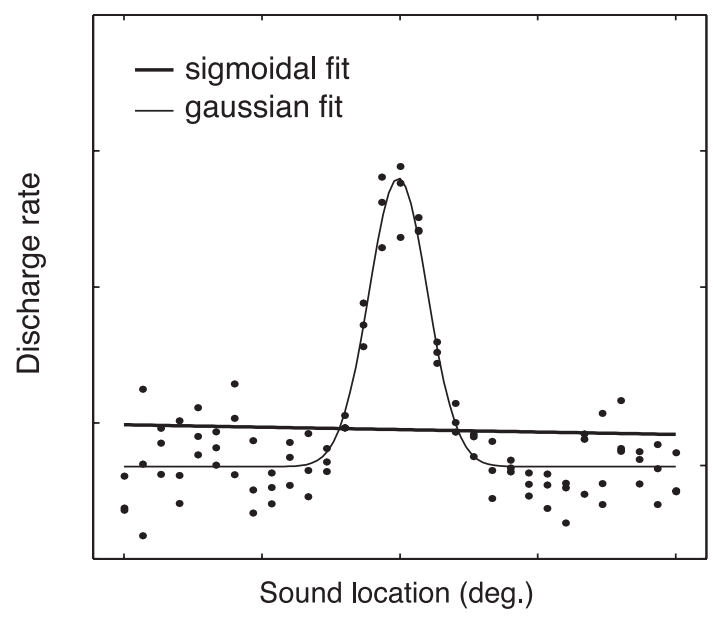

B

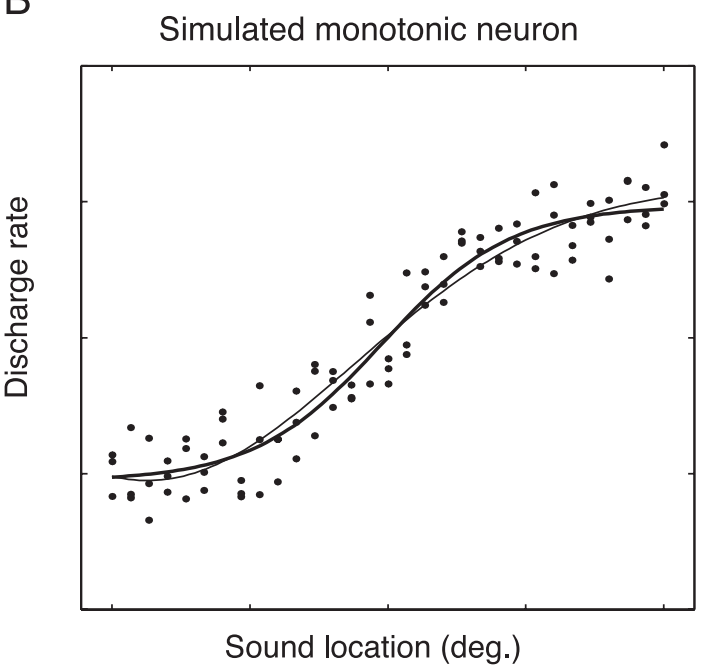

C

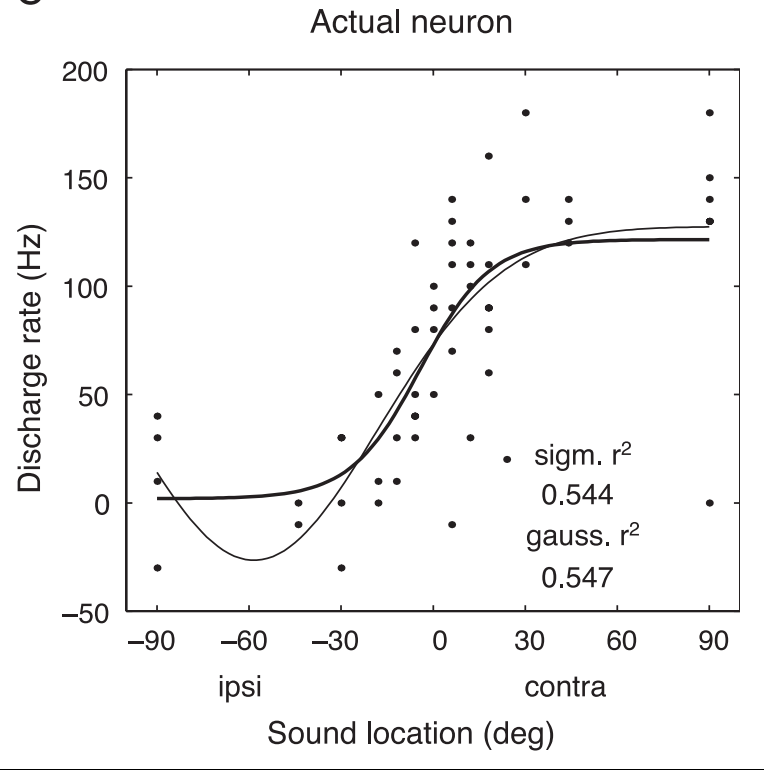


respond monotonically as a function of sound location, then the responses can potentially be fit nearly equally well by either a sigmoidal or a Gaussian function (Figure 3B). This equivalence occurs because Gaussians consist of two monotonic halves that can be very similar in shape to a sigmoidal function over a limited region of space. Thus, the hallmark of a monotonic code is not necessarily that sigmoids must fit better than Gaussians, but rather that Gaussians must not fit better than sigmoids. In short, we sought evidence for either of two possible scenarios: (1) that Gaussians consistently fit the azimuthal response functions better than sigmoids (or lines), indicating the presence of nonmonotonic sensitivity consistent with a place code for sound azimuth or (2) that sigmoidal and Gaussian fits be roughly equivalent to one another, indicating the presence of a monotonic sensitivity consistent with a rate code for sound azimuth.

The example neuron from Figure 2 has a clear monotonic sensitivity to sound location, and the response function could be fit by a sigmoid (thick trace), Gaussian (thin trace), and line (not shown) as illustrated in Figure 3C. All three fits were statistically significant $(p<.001)$. More importantly, only a monotonic portion of the Gaussian curve was needed to fit the responses, so that the sigmoid and Gaussian curves were nearly equally good-both accounting for $\sim 54-55 \%$ of the variance $\left(R^{2}=.544\right.$ for the sigmoid and .547 for the Gaussian). This neuron, then, clearly showed monotonic sensitivity to sound source azimuth in the frontal plane.

Figure 4 compares the linear, sigmoidal, and Gaussian fits across the entire population of 99 neurons. Figure $4 \mathrm{~A}$ gives a Venn diagram indicating the number of neurons for which linear, sigmoidal, and/or Gaussian fits were statistically significant $(p<.05)$. Fifty-two neurons (53\%) could be fit by all three functions (light gray region), whereas only three (3\%) were successfully fit by a Gaussian exclusively (dark gray region). The number of Gaussian-only fits is roughly the proportion predicted by chance with a criterion $p$ value of .05 . That sigmoids and Gaussians provided equally good fits is confirmed in Figure 4B, which compares the correlation coefficients $(R)$ for the sigmoidal fits with those of the Gaussian fits for all the neurons in the sample. The clustering of the points along a line of slope one indicates that Gaussians were rarely better than sigmoids. Therefore, the sensitivity for sound location among IC neurons appears to be overwhelmingly monotonic.

Indeed, the sigmoidal and Gaussian functions were remarkably similar in shape to each other over the frontal hemisphere. Figure 5A-I illustrates the neurons that had the second through eighth best sigmoidal and/or Gaussian fits (based on the correlation coefficients; only neurons tested over the full $180^{\circ}$ range of space were included; the neuron that had both the best Gaussian and the best sigmoidal fit is shown in Figures 2 and 3). The top eight sigmoidal fits and the top eight

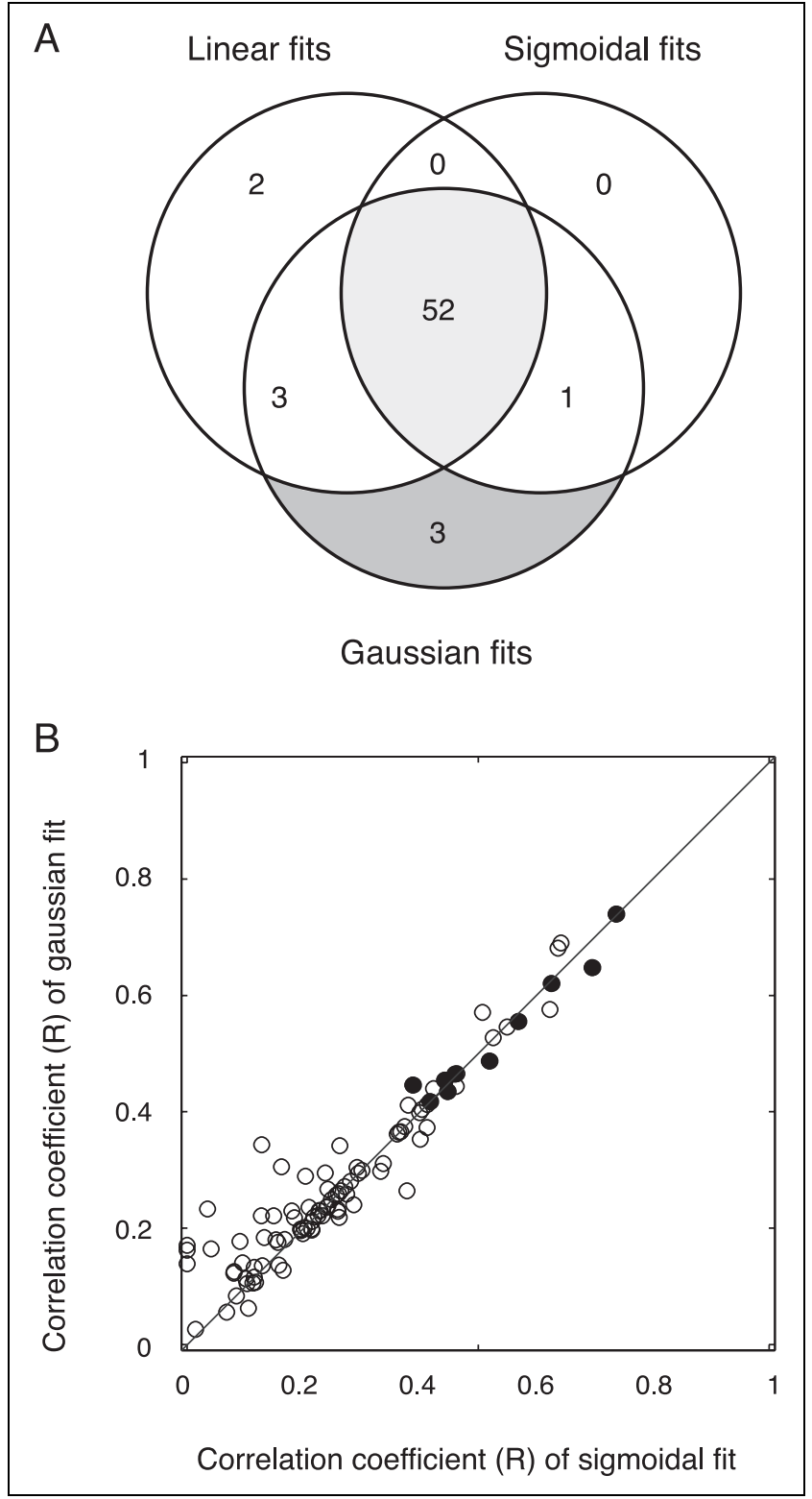

Figure 4. (A) Venn diagram of the relationship among the statistical significance of linear, sigmoidal, and Gaussian curve fits. All 99 neurons in the sample were included for this analysis. The two most relevant sets of neurons, those fit by all three functions and those fit by Gaussians only, are highlighted with light and dark gray, respectively. Curve-fitting and ANOVA were in good agreement with one another: 48 of the 49 cells showing a significant effect of sound location according to the ANOVA were also successfully fit by at least one function. (B) Relationship between the correlation coefficients $(R)$ of sigmoidal and Gaussian functions. The reference line has a slope of one. The filled circles indicate the neurons displayed in Figures 2, 3, 5A-I.

Gaussian fits amount to only 10 neurons total, which are identified with filled circles in Figure 4B. The sigmoidal and Gaussian functions were generally very similar to one another in shape: deviations between the functions were usually due to only one or two sound locations (e.g., Figure 5I) or occurred only in regions that were not sampled (e.g., Figure 5D). The $R^{2}$ values for the sigmoidal and Gaussian fits for these neurons were 
nearly identical to each other. In contrast, the quality of the fits for the three neurons fit only by the Gaussian was very poor. The curves and $R^{2}$ values for these neurons are shown in Figure $5 \mathrm{~J}-\mathrm{L}$. Thus, these Gaussian-only fits appear to reflect chance fluctuations in neural responsiveness rather than true nonmonotonic spatial sensitivity.
A great many mathematical functions could potentially be applied to fit these data, and the more free parameters involved, the better the fit that can in principle be achieved. Evidence that these linear, sigmoidal, and Gaussian functions were at least reasonably appropriate comes from the good correspondence between the neurons that were successfully fit by these
Figure 5. (A-I) The neurons with the second through eighth best sigmoidal and Gaussian fits. (Only neurons for which the entire $180^{\circ}$ range of space was sampled are included. The best neuron is shown in Figures 2 and 3.) Due to the similar quality of sigmoidal (thick lines) and Gaussian (thin lines) fits, the top eight selected from each category constitute a total of only 10 neurons. Dots indicate the means of all trials at the corresponding sound location; vertical bars represent standard error. Positive values on the $x$-axis indicate contralateral sound locations. The spontaneous activity prior to sound onset was subtracted from the stimulus-evoked activity for each trial in these plots. (J-L) The three neurons with significant Gaussian fits but insignificant monotonic (linear or sigmoidal) fits.
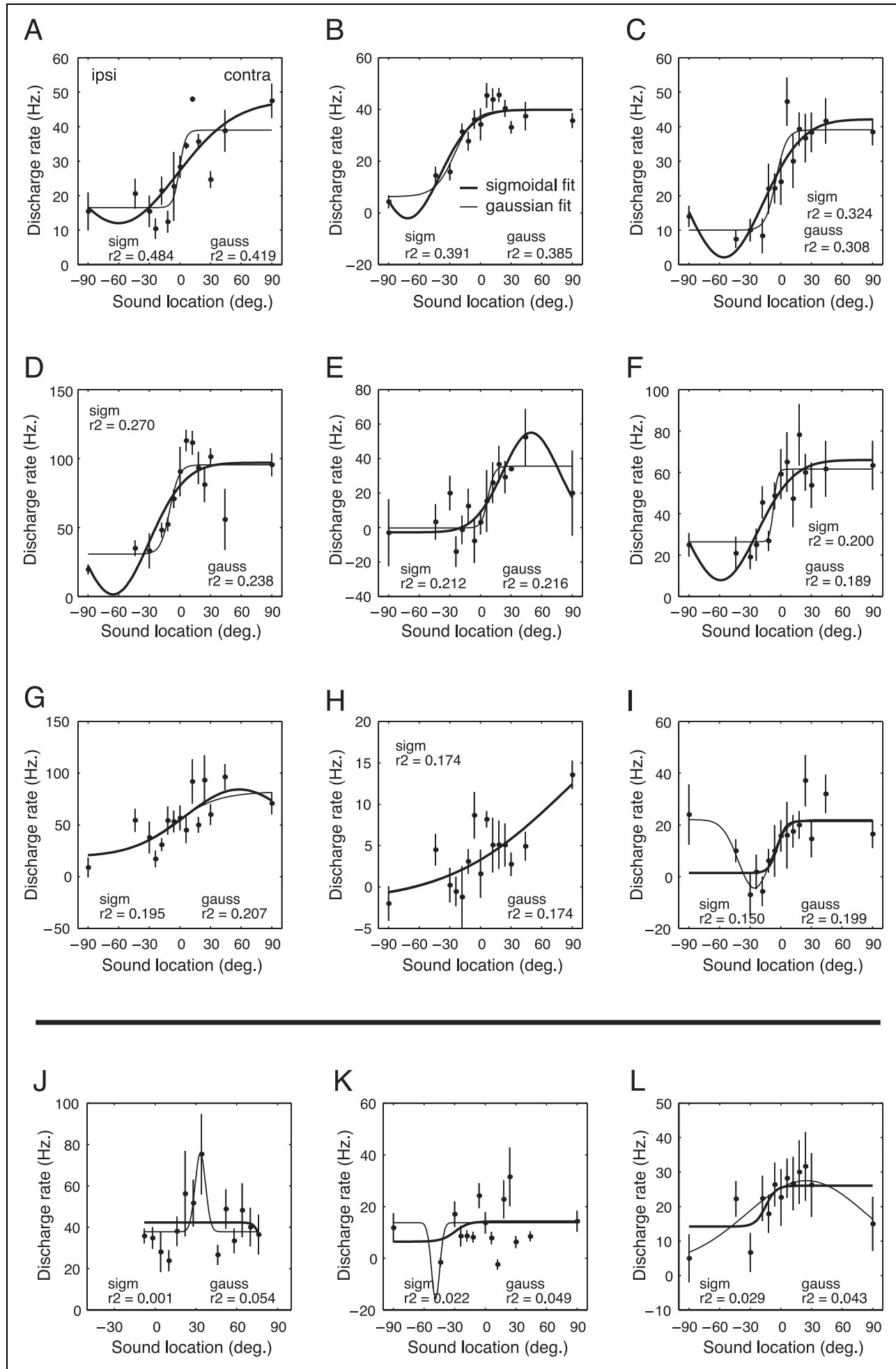
functions and neurons identified by ANOVA as having spatial sensitivity: 48 of the 49 neurons flagged by the ANOVA as being sensitive to sound location were also successfully fit by at least one function, and 46 were fit by all three. Thus, curve fitting rarely failed to detect spatial sensitivity when it was found by ANOVA.

\section{Distribution of Sigmoidal Inflection Points}

The family of statistically significant sigmoidal functions fit to the neurons in our sample is shown in Figure 6. Generally, the inflection points of these functions were clustered around the midline (although the slopes could vary substantially). A similar clustering of the region of steepest slopes to the midline has been observed in the ITD sensitivity curves of neurons in the guinea pig IC (McAlpine, Jiang, \& Palmer, 2001). The clustering of inflection points is consistent with, although not a requirement of, a rate code for sound location, whereas it is inconsistent with a place code, which calls for a distribution of spatial sensitivity profiles across the full expanse of space.

\section{Contralateral Bias}

A bias towards stronger responses for contralateral sound locations is readily apparent from the individual neurons shown in Figures 2, 3, and 5 as well as in the family of sigmoidal fits shown in Figure 6. The contralateral bias is illustrated for the entire population in Figure $7 \mathrm{~A}$, which shows the ratio of the mean responses to the most contralateral sound location tested divided by the mean responses to the most ipsilateral location.

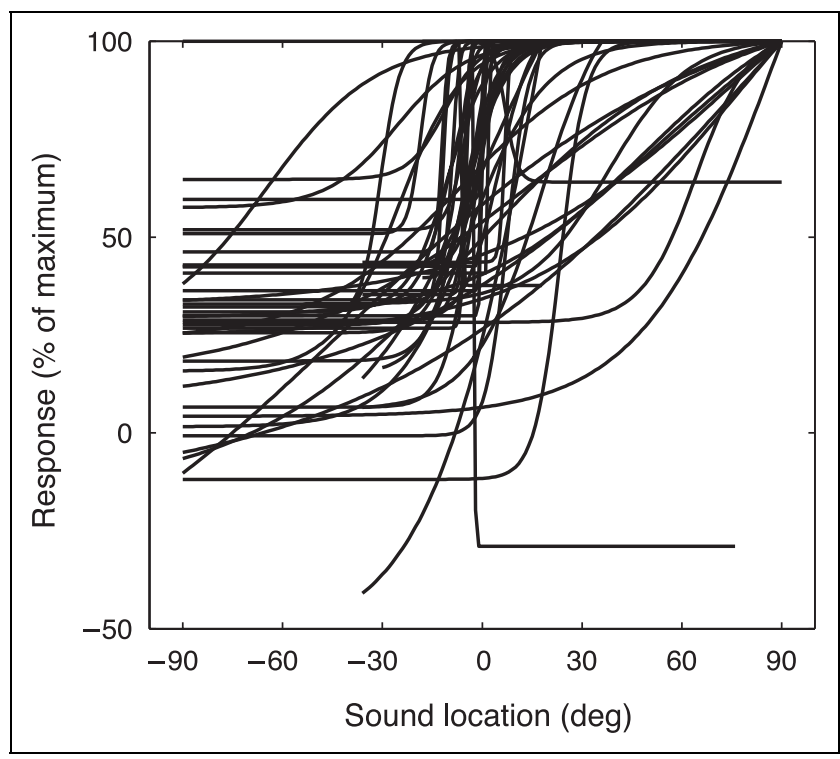

Figure 6. The family of statistically significant sigmoidal fits, normalized to the maximum response rate of each neuron. Note that the inflection points are clustered around the midline, but that the slopes can range from shallow to steep.

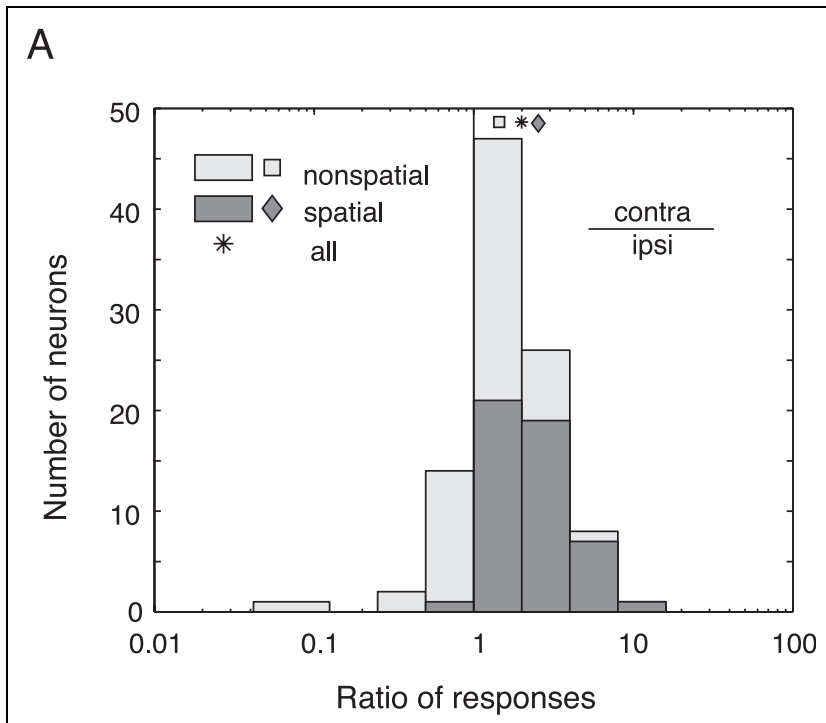

B

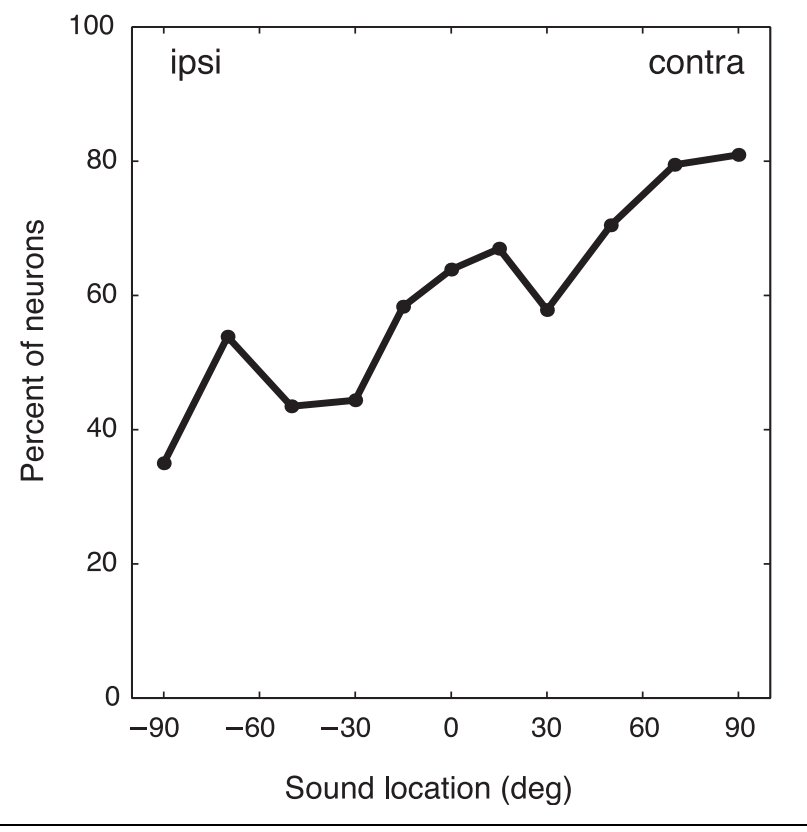

Figure 7. (A). Frequency histogram of the ratio of mean responses evoked by the most contralateral sound tested to the mean responses evoked by the most ipsilateral sound tested. The dark regions of the bars indicate the subset of neurons with statistically significant effects of sound location according to the ANOVA whereas the light regions indicate the spatially insensitive neurons. The mean ratios for the entire population, the spatially sensitive neurons, and the nonspatially sensitive neurons were 2.0, 2.6, and 1.4, respectively, and are indicated by the symbols at the top of the graph. To ensure that firing rates would be nonnegative for this analysis, the spontaneous activity was not subtracted. (B) Proportion of cells responding as a function of sound location. Cells were classified as responding to a sound location if the number of spikes during the response window after stimulus onset differed significantly from those during a comparable window of time before sound onset (paired $t$ test $p<.05$ ). Only sound locations for which at least 10 cells were tested with at least five trials each are included. All neurons, not just those with demonstrable spatial sensitivity, are included in this analysis. 
Table 1. Spatial Sensitivity of Different Neural Response Types

\begin{tabular}{|c|c|c|c|c|c|c|c|c|c|}
\hline \multirow{2}{*}{$\begin{array}{l}\text { (A) Temporal } \\
\text { response profile }\end{array}$} & \multirow[t]{2}{*}{ Cell Type } & \multicolumn{2}{|c|}{$N$ with Significant $A N O V A^{\mathrm{a}}$} & \multicolumn{2}{|c|}{$\begin{array}{c}\text { N Fit by All } \\
\text { Three Functions }^{\mathrm{a}}\end{array}$} & \multicolumn{2}{|c|}{$\begin{array}{c}\text { N Fit Only by } \\
\text { Gaussian }^{\mathrm{a}}\end{array}$} & \multirow{2}{*}{$\frac{N \text { Total }}{57}$} & \multirow{2}{*}{$\frac{\% \text { of Sample }}{58 \%}$} \\
\hline & & 31 & $54 \%$ & 32 & $56 \%$ & 2 & $4 \%$ & & \\
\hline & transient + sustained & 16 & $50 \%$ & 17 & $53 \%$ & 1 & $3 \%$ & 32 & $32 \%$ \\
\hline & inhibitory & 2 & $20 \%$ & 3 & $30 \%$ & 0 & $0 \%$ & 10 & $10 \%$ \\
\hline \multirow{2}{*}{$\begin{array}{l}\text { (B) Response } \\
\text { latency }\end{array}$} & latency $<$ median $(14 \mathrm{msec})$ & 24 & $56 \%$ & 24 & $56 \%$ & 1 & $2 \%$ & 43 & $48 \%$ \\
\hline & latency $\geq$ median & 22 & $48 \%$ & 25 & $54 \%$ & 2 & $4 \%$ & 46 & $52 \%$ \\
\hline \multirow[t]{3}{*}{$\begin{array}{l}\text { (C) Frequency } \\
\text { response range }\end{array}$} & $\begin{array}{l}\text { responsive to low tones } \\
\text { only }(\leq 1000 \mathrm{~Hz})\end{array}$ & 11 & $55 \%$ & 12 & $60 \%$ & 0 & $0 \%$ & 20 & $32 \%$ \\
\hline & $\begin{array}{l}\text { responsive to high and low } \\
\text { or high only }(\geq 2000 \mathrm{~Hz})\end{array}$ & 21 & $70 \%$ & 20 & $67 \%$ & 1 & $3 \%$ & 30 & $48 \%$ \\
\hline & unresponsive to tones $^{c}$ & 2 & $15 \%$ & 4 & $31 \%$ & 1 & $8 \%$ & 13 & $21 \%$ \\
\hline \multirow{3}{*}{$\begin{array}{l}\text { (D) "Best" } \\
\text { frequency }\end{array}$} & low $(\leq 1000 \mathrm{~Hz})$ best frequency & 27 & $57 \%$ & 29 & $62 \%$ & 1 & $2 \%$ & 47 & $75 \%$ \\
\hline & high $(\geq 2000 \mathrm{~Hz})$ best frequency & 3 & $50 \%$ & 2 & $33 \%$ & 1 & $17 \%$ & 6 & $10 \%$ \\
\hline & unresponsive to tones ${ }^{c}$ & 4 & $40 \%$ & 4 & $40 \%$ & 0 & $0 \%$ & 10 & $16 \%$ \\
\hline
\end{tabular}

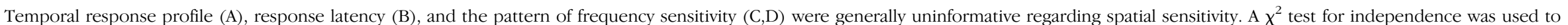

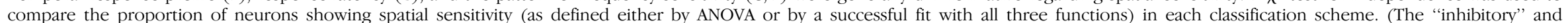

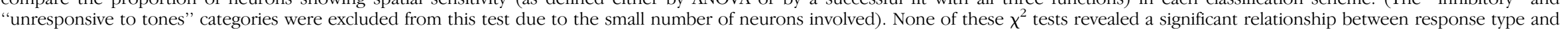
spatial sensitivity.

aThe percentages in columns 2, 4, and 6 reflect the numbers from columns 1, 3, and 5 divided by the number of neurons in that response category (column 7)

${ }^{\mathrm{b}}$ The percentages in column 8, labeled "\% of sample," reflect the proportion of the total sample (or tested subsample) that makes up each response category.

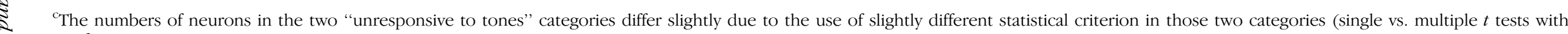
Bonferroni correction). 
On average, neurons fired about twice as vigorously to the most contralateral location compared with the most ipsilateral location. The bias for contralateral locations was, not surprisingly, most profound in the population of neurons that showed a statistically significant impact of sound location (by ANOVA, dark regions).

\section{Breadth of Spatial Sensitivity}

The spatial sensitivity of our sample of IC neurons is clearly very broad. The individual neuron in Figures 2 and 3 responded to at least half of the sound locations tested. Figure 7B illustrates the "point image" of activity in the entire population of IC neurons as a function of sound location. All 99 neurons were included in this analysis. The proportion of neurons that showed statistically significant responses to sounds increased from a low of about $30 \%$ for the most ipsilateral sound location to a high of about $80 \%$ for the most contralateral location.

\section{Response Characteristics of Spatially Sensitive Neurons}

The population of neurons that were sensitive to sound location was composed of neurons with a broad range of other response characteristics (Table 1). Both neurons with primarily transient responses and those with sustained activity in addition to transient responses could be spatially sensitive, as determined either by ANOVA or by function fitting. Neurons with inhibitory responses to sound could also be spatially sensitive. The proportions of neurons showing spatial sensitivity did not differ among these groups $\left(\chi^{2}, p>.05\right)$. No differences in the proportions of spatial sensitivity in neurons with response latencies $<14 \mathrm{msec}$ (the median latency) and those with response latencies $\geq 14$ msec were observed. The mean response latency of the neurons in our sample was $\sim 12$ msec.

Frequency sensitivity is a response characteristic that might be expected to have relevance to spatial sensitivity because neurons sensitive to low frequencies have been specifically implicated in the processing of ITDs, whereas neurons sensitive to high frequencies are thought to mediate primarily ILDs. Indeed, best frequency and best ITD have been found to be correlated in guinea pig IC neurons (McAlpine, Jiang, \& Palmer, 1996; McAlpine et al., 2001). However, the relationship between frequency sensitivity and binaural difference cues is not an exclusive one, as high best-frequency neurons have also been found to be sensitive to ITDs in the amplitude-modulated waveform of high-frequency tones (e.g., Yin, Kuwada, \& Sujaku, 1984). Additionally, in monkeys, ILD cues remain quite sizeable even at reasonably low frequencies $(\sim 15-$ $25 \mathrm{~dB}$ SPL at $2 \mathrm{kHz}$; Spezio, Keller, Marrocco, \& Takahashi, 2000), raising the possibility that ILD sensitivity could also be mediated by low frequency neurons.
We assessed the responses to tones of fixed intensity of 63 of the neurons in our sample. We classified the frequency sensitivity of the population of neurons in two ways, based on either the range of tones evoking significant responses or the single tone that evoked the most vigorous response, with respect to the approximate low and high frequency ranges in which ITDs and ILDs are thought to be most effective (Table 1).

In the first classification scheme, we categorized neurons as responsive to low frequencies if they exhibited a statistically significant response ( $t$ test) to at least one tone of frequency $\leq 1000 \mathrm{~Hz}$; those sensitive to high frequencies were those that exhibited statistically significant responses to frequencies $\geq 2000 \mathrm{~Hz}$. The criterion for a significant response was a $p$ value $<.05$, after Bonferroni correction for performance of multiple tests. Some neurons did not exhibit significant responses to tonal stimuli in the frequency range and sound level that we tested. We classified such neurons as unresponsive to tones. Table 1 shows the proportions of neurons in each category. There were no differences in the proportions of neurons in these categories showing statistically significant effects of sound location $\left(\chi^{2}, p>.05\right)$.

In the second classification scheme, we classified neurons as having a low or high best frequency if the frequency that elicited the maximum response was $\leq 1000$ or $\geq 2000 \mathrm{~Hz}$. Again, if the maximum response was not statistically significant ( $t$ test, criterion $p$ value $<.05$, no Bonferroni correction), the neuron was classified as unresponsive to tones. This second scheme is likely to bear a closer relationship to the traditional metric of characteristic frequency measured at threshold, although the correspondence may be inexact (cf. Ramachandran, Davis, \& May, 1999; Rose, Greenwood, Goldberg, \& Hind, 1963; see also Discussion). Again, there were no significant differences in the proportions of neurons showing effects of sound location as a function of frequency (Table $1, \chi^{2}, p>.05$ ).

As Table 1 shows, the largest classes of neurons were those that responded broadly to both low and high frequencies (in the first classification scheme) and those that exhibited their maximum responses to low frequency tones (in the second classification scheme). A similar bias towards low characteristic frequencies is evident in a previous study in rhesus monkey IC (Ryan \& Miller, 1978; see also Discussion).

\section{DISCUSSION}

\section{Summary of Findings}

Our findings confirm those of previous studies in other species in showing that a sizeable population of neurons in the primate IC carries information regarding the locations of sounds. Neural responses tended to increase monotonically for sounds located more contralaterally. No clearly bounded ranges of best locations within the 
frontal hemisphere were in evidence. The bias for the contralateral hemifield is perhaps the strongest evidence that the information contained within the responses of IC neurons would in fact be suitable for representing the locations of sounds in space: although the sensitivity of neurons was broad and responses were variable, the population of active neurons was clearly larger and discharge rates were higher in the IC contralateral to the location of the sound than they were in the ipsilateral IC.

\section{Comparison with Previous Studies}

The contralateral bias in primate IC is the most consistent point of comparison with previous studies in other species, having been reported in nearly every previous study. Evidence concerning the existence, or relative proportions, of monotonic versus nonmonotonic coding for sound location in the IC of other species is more difficult to evaluate. Studies of ITD sensitivity show that the distribution of best ITDs spans, but can also extend well beyond, the range of ITDs that occur under natural conditions and is therefore partially consistent with both monotonic and nonmonotonic coding of azimuth (McAlpine et al., 2001; Fitzpatrick, Batra, Stanford, \& Kuwada, 1997; Stanford, Kuwada, \& Batra, 1992; Palmer, Rees, \& Caird, 1990; Caird \& Klinke, 1987; Chan, Yin, \& Musicant, 1987; Yin, Chan, \& Irvine, 1986; Kuwada \& Yin, 1983). The literature on ILD sensitivity also contains examples of both monotonic and nonmonotonic functions (Fuzessery, Wenstrup, \& Pollak, 1990; Irvine, 1987; Irvine \& Gago, 1990; Semple \& Kitzes, 1987; Calford, Moore, \& Hutchings, 1986; Stillman, 1972; Benevento \& Coleman, 1970) in the range of naturally occurring ILDs (e.g., Hartung \& Sterbing, 2001; Spezio et al., 2000; Brugge et al., 1994; Fuzessery et al., 1990; Musicant, Chan, \& Hind, 1990; Calford et al., 1986; Harrison \& Downey, 1970).

Free-field studies also provide conflicting evidence. Topographically organized receptive fields have been found in the external nucleus of guinea pig and barn owl IC (Binns et al., 1992; Knudsen \& Konishi, 1978), but a similarly clear-cut case has not emerged from a wealth of experiments in cat IC. Semple, Moore, Aitkin, and colleagues assessed the spatial sensitivity of cat IC neurons and noted that the region of highest responsiveness usually included the acoustical axis of the contralateral ear (Moore, Hutchings, et al., 1984; Moore, Semple, et al., 1984; Semple et al., 1983). Both monotonic and nonmonotonic azimuthal sensitivities in frontal space were reported by Aitkin and colleagues in several later studies (Aitkin et al., 1984, 1985; Aitkin \& Martin, 1987).

In a more recent cat study using virtual space methods, Delgutte et al. (1999) distinguished between contrapreference units, defined as "those for which the response falls $<50 \%$ of maximum on the ipsilateral side of the best azimuth but remains $>50 \%$ of the maximum on the contralateral side," and tuned units, defined as "those with responses that fall $<50 \%$ of the maximum on both sides of the best azimuth." The definition of contrapreference does not preclude nonmonotonic responses, and their illustrated examples include both monotonic and nonmonotonic neurons. The definition of "tuned units" does correspond to azimuthal nonmonotonicity, and such units made up about a third of their sample, with best azimuths smoothly distributed over the frontal contralateral $0-54^{\circ}$. In short, substantial populations of both nonmonotonic and monotonic azimuthal response functions were reported in their study.

On the whole, our results suggest that primates are similar to cats and other mammals in that monotonic sensitivity to sound azimuth exists in the IC of all of these species. The primate appears to differ from the cat, guinea pig, and barn owl IC in that primate IC apparently does not also contain notable nonmonotonic sensitivity to sound azimuth. (Of course, our study does not address the coding of sound source elevation, the coding of sound locations behind the animal, nor the relative contributions of interaural timing differences, level differences, and spectral cues to the pattern of spatial sensitivity reported here. These important issues have been explored in other species, and await further research in primates.)

The absence of nonmonotonic azimuthal sensitivity in our study compared with some previous studies might represent a species difference, although differences between the methods used could contribute to the differences in the findings, especially since not all previous studies have found substantial nonmonotonic azimuthal sensitivity. The most obvious general difference is the awake state of our animals compared to the use of anesthetized animals in most previous studies. Other important methodological differences include the statistical analyses employed here to distinguish monotonic from nonmonotonic azimuthal sensitivity: it is possible that some of the nonmonotonic azimuthal sensitivity evident in other studies might not meet our requirements for nonmonotonicity.

There may also be differences in the region of the IC sampled most thoroughly. In our study, the preponderance of neurons responding to low frequency sounds suggests that the shallower low-frequency regions of the IC may have predominated in our sample. Thus, it is possible that substantial nonmonotonic sensitivity to sounds may yet be uncovered at deeper locations. However, the low-frequency bias in our sample may also reflect a true feature of the representation of frequency in macaque IC, as a similar bias is evident in a previous study in awake rhesus monkeys (Ryan \& Miller, 1978).

Finally, while it would be overreaching to rule out the possible presence of some nonmonotonic spatial sensitivity under conditions different from those employed here, it seems clear from our findings that the kind of nonmonotonic sensitivity needed to form a place code for sound location in the primate IC is unlikely to 
emerge. To be useful, such a place code should be capable of representing the locations of sounds in the frontal plane (and elsewhere), regardless of sound level or frequency. A sizeable population of neurons, with receptive fields distributed across the sampled region of space, would be needed. We found no compelling evidence of such a representation.

\section{Implications for the Read-Out Mechanism and Integration with Visual Information}

How might the representation of sound location in the IC be "read out" to produce a percept of sound location and guide sound localizing behavior? The advantage of a rate code is that at least in theory, a single individual neuron with broad monotonic sensitivity to sound location can provide an ideal observer with sufficient information to determine the location of a sound source via a one-to-one correspondence between the level (and/or pattern) of neural activity and sound location. Indeed, Middlebrooks, Xu, Eddins, and Green (1998) have provided elegant demonstrations of this possibility in cat auditory cortical neurons.

In practice, however, several problems interfere with this theoretical extreme and suggest that the perception of sound source location must certainly rely on the activity of a population of neurons. First, individual neurons have variability in their discharge levels. Noisy discharge levels will produce noisy behavior unless error checking is performed by some kind of comparison across the population of space-sensitive neurons. Second, neural responses depend on sound parameters other than location: most notably, sound frequency and pressure level. Reliance on the activity of a single neuron to determine sound location would cause sounds of different frequencies or intensities arising from the same location to be attributed to different locations. Finally, a single neuron cannot simultaneously convey separate information concerning more than one distinct sound source. These problems have long been recognized as important, but they are particularly acute for rate codes. In contrast, place codes use the identity of the active population(s) to encode the location of one or more stimuli, and multiple simultaneous locations can be represented using multiple peaks of activity. Variations in the level of activity within the active population(s) do not generally have devastating effects on performance (for a discussion, see Groh, 2001).

Is a place code for auditory space ultimately created from the representation of space found in the primate IC? Such a transformation cannot add resolution or accuracy over that possible based on signals present early in the auditory pathway, and thus might not be necessary at all were it not for the advantages to be accrued from merging auditory information with visual information. Vision influences the perceived locations of sounds, yet visual spatial information is initially encoded in both a format and a frame of reference that differs from auditory spatial information. Considerable evidence links the IC with visual-auditory integration. Vision exerts control over the spatial sensitivity of neurons in the external nucleus of the barn owl IC (e.g., Brainard \& Knudsen, 1993). Projections from retina to the pericentral nucleus of the IC have been reported in rat and monkey (Itaya \& Van Hoesen, 1982) and visually responsive neurons have been identified in this subnucleus in cat (Mascetti \& Strozzi, 1988). We have previously shown that the position of the visual sense organ, the eye, affects the responses of many neurons in IC. This eye position influence may be part of an effort by the brain to bring auditory and visual signals together (Groh et al., 2001).

How might a place code for sound location be created from the rate code found in the IC? Two candidate circuits are illustrated in Figure 8. The first involves combining signals from the IC on opposite sides of the brain in an additive fashion (Figure 8A) while the second option involves combining sigmoidal signals with different inflection points from IC neurons on the same side of the brain in a subtractive fashion (Figure 8B; see Groh \& Sparks, 1992).

Either method could, in principle, create neurons with tuning for sound location, although there are thorny issues for both possibilities. For example, unilateral lesions of cat IC produce only contralateral deficits in sound localization abilities (Jenkins \& Masterton, 1982). These findings argue against the opposite-sides model (Figure 8A) because eliminating the input from one side of the brain in this model would disrupt the receptive fields across all of space, not just the contralateral field. A problem for both models is that they would seem to require a distribution of inflection points across the contralateral field, whereas the inflection points in our sample tended to cluster near the midline. Details such as how the candidate component neurons of such circuits change their spatial tuning as a function of sound level will also be important for helping to distinguish between these possibilities (cf. Gaese \& Johnen, 2000; Blatchley \& Brugge, 1990; Ehret \& Merzenich, 1988; Irvine, 1987; Aitkin et al., 1984, 1985; Aitkin, 1991; Aitkin, Tran, \& Syka, 1994; Irvine \& Gago, 1990; King \& Hutchings, 1987; Semple \& Kitzes, 1987; Calford et al., 1986; Wise \& Irvine, 1985; King \& Palmer, 1983; Ryan \& Miller, 1978; Hind, Goldberg, Greenwood, \& Rose, 1963). In short, these models merely illustrate two possible circuits that could in principle perform a conversion from a rate to a place code in a relatively straightforward fashion, and additional details will be needed to identify which (if any) of these possibilities is likely.

\section{Conclusions}

Given that sound location must be computed from binaural and spectral cues, it is not surprising that the format employed in the primate IC differs from that 
Figure 8. Two possible circuits for converting monotonic spatial sensitivity to nonmonotonic spatial sensitivity. The circuit in (B) is based on a component of the vector subtraction model of Groh and Sparks (1992).

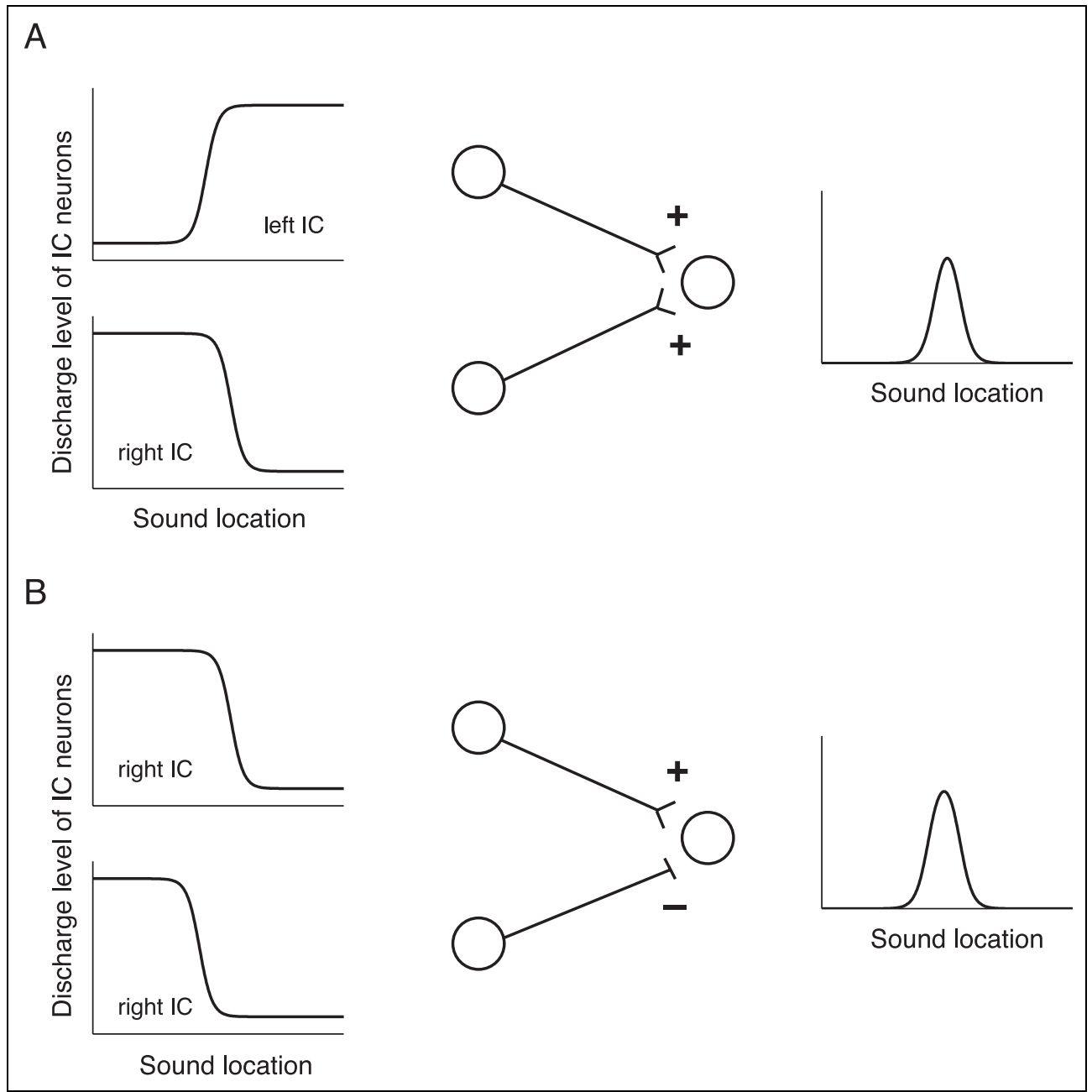

used in sensory systems, such as vision or touch where stimulus location can be inferred from the location of active receptors on the sensory epithelia. However, the differences in format do pose problems for the eventual convergence of sensory information from different modalities. The auditory signals in the IC carry information about both the location of the stimulus and the orientation of the eyes (Groh et al., 2001), but it appears that the representation of this information requires both a transformation into the proper format as well as into the proper reference frame before it is suitable for eventual integration with visual signals.

\section{METHODS}

\section{Animal Preparation and Behavioral Task}

Three adult female rhesus monkeys served as subjects for this experiment. All procedures were approved by the Institutional Animal Care and Use Committee at Dartmouth. Animals underwent a surgery under isoflurane anesthesia and aseptic conditions to implant a head post for restraining the head and a scleral eye coil for monitoring eye position at $500 \mathrm{~Hz}$ (Judge, Richmond, \&
Chu, 1980; Robinson, 1963). They were then trained to maintain fixation (within $\pm 6^{\circ}$ ) of a light emitting diode (LED) located in front of the animal. Five hundred milliseconds after the animal began fixating, one or fourÂsounds (500 or $1000 \mathrm{msec}$ duration, 500-msec interstimulus interval, $\sim 2$ - to 4-sec intertrial interval) were presented. Trials were terminated and discarded from the analysis if the monkey failed to maintain fixation throughout the trial. Different sound locations (detailed below) were randomly interleaved on successive stimulus presentations both within an individual trial and on successive trials. During the recording of some neurons, different fixation positions were randomly interleaved on successive trials. The data for these eccentric fixation positions were excluded from the analyses herein.

\section{Acoustic Environment and Stimuli}

These experiments were conducted under free-field conditions with sound-reflecting surfaces and obstructions limited to those necessary for presenting stimuli, monitoring eye position, and restraining the animal. 
Echo-absorbent material lined the walls, ceiling (3-in painted Sonex One), as well as the floor (carpet). The latter 74 experiments were conducted in a single-walled sound attenuation chamber (IAC). The first 25 experiments were conducted in a room with concrete walls and plaster ceiling, also lined with Sonex foam, and floor covered with sound-absorbing rubber mats. Obstructions and reflective surfaces in the environment consisted of a wooden or plastic cube-shaped frame supporting the field coil (outer dimensions 2 or $3 \mathrm{ft}$ on a side, composed of $1.5 \times 2.75$-in. supports), a $72 \times$ 48-in. peg board containing the LEDs hanging on the wall, and the primate chair constructed of Lexan plastic and aluminum. The attachment of the head post to the primate chair lay above and/or behind the path of the sounds to the monkey's ears. The alternate paths taken to reach the ears by sounds reverberating off these structures could, in theory, create peaks in spatial sensitivity functions that were actually monotonic: an erroneously high (or low) response could be attributed to a particular sound location if its echo were to arrive from a more (or less) favorable direction. If spatial sensitivity were nonmonotonic, the presence of echoes could create multiple peaks or cause a discrepancy between the measured location of the receptive field and its true location. However, it seems unlikely that echoes could systematically make nonmonotonic spatial sensitivity appear monotonic. Furthermore, the presence of any echoes or distortions in our sound field were evidently not sufficient to disrupt sound localization behavior: in the course of other experiments, we have successfully trained three monkeys to make saccades to the locations of the loudspeakers in our apparatus (Metzger et al., 2003). Thus, the conditions we used were sufficient to produce cues to sound location that the monkeys could use appropriately.

Sounds were presented from any of 15 different speakers (Audax TWO25V2) positioned between $-90^{\circ}$ and $90^{\circ}$ (negative numbers indicate ipsilateral locations throughout this article) along the horizontal meridian at a distance of 48-74 in. from the animal (along the perimeter of the testing chamber). Speakers could be mounted in a range of positions. The most typical set of positions was $\pm 90, \pm 44, \pm 30, \pm 24, \pm 18, \pm 12, \pm 6$, and 0 . Forty-three neurons were tested with this set of locations. Twenty-six neurons were tested with speakers spaced every $20^{\circ}$ over $160-180^{\circ}$ of the frontal hemifield. An additional nine neurons were tested with speakers spanning $72-114^{\circ}$ of the contralateral frontal hemifield, and 21 neurons were tested with speakers spanning 36$66^{\circ}$ of space centered on the midline or in the contralateral hemifield. These latter two categories included seven neurons with detailed ( $6^{\circ}$ sampling) from $-8^{\circ}$ to $76^{\circ}$ (five neurons) and from $22^{\circ}$ to $76^{\circ}$ (two neurons). When only the neurons tested across the full $180^{\circ}$ range were included $(n=63)$, no substantive differences in the results were noted. On average, $10.6 \pm 7.9(S D)$ repetitions of each stimulus condition were conducted for each neuron.

The auditory stimulus was a broadband white noise ( $\sim 500 \mathrm{~Hz}$ to $\sim 18 \mathrm{kHz}$ - the range in which the speakers performed well). The level of this sound was $53 \mathrm{~dB}$ SPL (RMS) when measured with a sound level meter (Bruel \& Kjaer, model 2237 integrating sound level meter with model 4137 condenser microphone) placed at the location normally occupied by the animal's head. The gain of the analog signal delivered to each speaker was adjusted to compensate for differences in the gain of each speaker and/or distance from the animal to an accuracy of $\pm 2 \mathrm{~dB}$ SPL. The maximum standard deviation in the level of any individual frequency component was $<2 \mathrm{~dB}$ across the different speakers (measured with Bruel \& Kjaer type 4190 free field 0.5 -in. microphone with type 2669 preamplifier and type 2804 power supply). For 63 of the 99 experiments, responses to tonal stimuli (typically $400 \mathrm{~Hz}$ to $12 \mathrm{kHz}$ at $55 \mathrm{~dB}$ SPL) were also recorded after testing of sound location sensitivity had been terminated. No behavioral task was required of the animal for these trials. These tonal stimuli were delivered from a single speaker location, usually the $90^{\circ}$ contralateral speaker.

\section{Recording}

Once the animals were trained, a cylinder was implanted using stereotaxic techniques in an aseptic surgical procedure under isoflurane anesthesia. Recordings were made from the left IC in one monkey, the right IC in the second, and from both IC in the third. Electrodes approached the IC at an angle of $33^{\circ}$ lateral from vertical in the coronal plane. Search stimuli consisted of broadband noise delivered from any of the speaker positions in use that day. Once the electrode had reached the IC (as determined by the advent of acoustic responses in the multiunit activity), any neuron with isolatable action potentials was accepted for further study, without regard to what type of spatial sensitivity the neuron appeared to have. Standard recording techniques were employed: electrical potentials were amplified and action potentials were detected using a window discriminator (Bak Electronics). The time of occurrence of action potentials was stored for off-line analysis. The number of action potentials occurring within a specified time window following stimulus onset was counted. Because individual cells showed various combinations of phasic and sustained responses (Ryan \& Miller, 1977), this time window was chosen by inspection of each neuron's unsmoothed PSTH including all trials and all sound locations to best capture the response period. Response windows ranged from the first $50 \mathrm{msec}$ after stimulus onset for very phasic cells to the first $500 \mathrm{msec}$ for tonic or inhibitory cells. On the whole, choice of response window did not influence 
the overall pattern of results, which was similar when the whole $500 \mathrm{msec}$ response period was used. Unless otherwise noted, the baseline firing rate for a corresponding period before the onset of the first stimulus on each trial was subtracted from each stimulus response period for that trial.

The locations of our recordings were confirmed by visualizing an electrode approaching the IC of each monkey via MRI at the Dartmouth Brain Imaging Center (see Groh et al., 2001, for details). Using these techniques, we were able to discern both the electrode and the morphological features of the brain. The position of the electrode with respect to the IC was readily apparent, though finer distinctions such as the locations of the subnuclei of the IC were not distinguishable using this method. Consequently, all our recording locations are lumped together under the rubric of IC. The penetrations in which recordings were made spanned an 8- to $9-\mathrm{mm}^{2}$ range of the cylinder grid in two of the four IC, and somewhat smaller regions in the other IC. We also noted the depth of the recordings with respect to the point of entry into the IC. These depths ranged from 0 to $\sim 3.4 \mathrm{~mm}$. Especially for later recording sessions, these numbers may substantially underestimate the true depth of the recordings as it becomes increasingly difficult to discern the point of entry into the IC over time.

\section{Data Analysis}

\section{Function Fitting}

We assessed monotonicity of spatial tuning by evaluating how well functions that are solely monotonic (lines and sigmoids) compared with those that are nonmonotonic but contain monotonic regions (Gaussians) at capturing the relationship between discharge rate and sound location. Linear fits were conducted with conventional regression methods. The nonlinear fitting of sigmoids and Gaussians was performed in Matlab (Mathworks) using the "lsqnonlin" function, which conducts an iterative search to find the parameters yielding the best fit to the data.

\section{Assessment of Response Latency}

Response latency was determined from the PSTH of each neuron. The unsmoothed PSTH was constructed using a 3-msec bin width. The mean and standard deviation of the firing rate in the bins during the baseline period before the onset of the first stimulus was determined. The first bin after stimulus onset in which the discharge rate exceeded the baseline firing rate plus three times the standard deviation of the baseline firing rate was taken as the response latency of the neuron. Neurons whose responses were solely inhibitory or those in which the response latency as measured in this fashion exceeded $400 \mathrm{msec}$ were excluded from the analysis.

\section{Acknowledgments}

We thank Uri Werner-Reiss for suggesting one of the models in Figure 8. We also thank Kimberly Rose Clark and Amanda S. Trause, who assisted in the collection of the data for this study, and Yale Cohen, Ryan Metzger, O'Dhaniel Mullette-Gillman, Alan Palmer, and Uri Werner-Reiss, who provided helpful comments on this manuscript. We are indebted to Souheil Inati and Tammy Laroche of the Dartmouth Brain Imaging Center for assistance with MRI. We are grateful for financial support from the following sources: the Alfred P. Sloan Foundation, the McKnight Endowment Fund for Neuroscience, the Whitehall Foundation, the John Merck Scholars Program, the Office of Naval Research Young Investigator Program, the EJLB Foundation, The Nelson A. Rockefeller Center at Dartmouth, and NIH NS 17778-19 (J.M.G.) and NIH NS 44666-01 (K.A.K.).

Reprint requests should be sent to Jennifer M. Groh, Department of Psychological and Brain Sciences, Dartmouth College, Room 356, 6207 Moore Hall, Hanover, NH 03755, USA, or via email: jennifer.m.groh@dartmouth.edu.

\section{Note}

1. Naturally, because sound location is a spherical parameter, monotonicity is not expected of a rate code if the sampling of sound location wraps around the head so that both front and back locations with the same interaural timing and level differences are included. Indeed, a more precise formulation of the rate code hypothesis is that discharge rates should vary monotonically with the sine of the azimuthal angle rather than the azimuthal angle itself. For this reason, we limited our explorations to sound locations along the frontal horizontal plane and we investigated the shape of the response functions of IC neurons to sounds within this region of the auditory environment.

\section{REFERENCES}

Aitkin, L. (1991). Rate-level functions of neurons in the inferior colliculus of cats measured with the use of free-field sound stimuli. Journal of Neurophysiology, 65, 383-392.

Aitkin, L., Tran, L., \& Syka, J. (1994). The responses of neurons in subdivisions of the inferior colliculus of cats to tonal, noise and vocal stimuli. Experimental Brain Research, 98, 53-64.

Aitkin, L. M., Gates, G. R., \& Phillips, S. C. (1984). Responses of neurons in inferior colliculus to variations in sound-source azimuth. Journal of Neurophysiology, 52, 1-17.

Aitkin, L. M., \& Martin, R. L. (1987). The representation of stimulus azimuth by high best-frequency azimuth-selective neurons in the central nucleus of the inferior colliculus of the cat. Journal of Neurophysiology, 57, 1185-1200.

Aitkin, L. M., Pettigrew, J. D., Calford, M. B., Phillips, S. C., \& Wise, L. Z. (1985). Representation of stimulus azimuth by low-frequency neurons in inferior colliculus of the cat. Journal of Neurophysiology, 53, 43-59.

Benevento, L. A., \& Coleman, P. D. (1970). Responses of single cells in cat inferior colliculus to binaural click stimuli: Combinations of intensity levels, time differences and intensity differences. Brain Research, 17, 387-405.

Binns, K. E., Grant, S., Withington, D. J., \& Keating, M. J. 
(1992). A topographic representation of auditory space in the external nucleus of the inferior colliculus of the guinea-pig. Brain Research, 589, 231-242.

Blatchley, B. J., \& Brugge, J. F. (1990). Sensitivity to binaural intensity and phase difference cues in kitten inferior colliculus. Journal of Neurophysiology, 64, 582-597.

Bock, G. R., \& Webster, W. R. (1974). Coding of spatial location by single units in the inferior colliculus of the alert cat. Experimental Brain Research, 21, 387-398.

Brainard, M. S. (1994). Neural substrates of sound localization. Current Opinions in Neurobiology, 4, 557-562.

Brainard, M. S., \& Knudsen, E. I. (1993). Experience-dependent plasticity in the inferior colliculus: A site for visual calibration of the neural representation of auditory space in the barn owl. Journal of Neuroscience, 13, 4589-4608.

Brugge, J. F., Reale, R. A., Hind, J. E., Chan, J. C., Musicant, A. D., \& Poon, P. W. (1994). Simulation of free-field sound sources and its application to studies of cortical mechanisms of sound localization in the cat. Hearing Research, 73, 67-84.

Caird, D., \& Klinke, R. (1987). Processing of interaural time and intensity differences in the cat inferior colliculus. Experimental Brain Research, 68, 379-392.

Calford, M. B., Moore, D. R., \& Hutchings, M. E. (1986). Central and peripheral contributions to coding of acoustic space by neurons in inferior colliculus of cat. Journal of Neurophysiology, 55, 587-603.

Chan, J. C., Yin, T. C., \& Musicant, A. D. (1987). Effects of interaural time delays of noise stimuli on low-frequency cells in the cat's inferior colliculus: II. Responses to band-pass filtered noises. Journal of Neurophysiology, 58, 543-561.

Churchland, P. S., \& Sejnowski, T. J. (1992). The computational brain. Cambridge: MIT Press.

Clarey, J. C., Barone, P., \& Imig, T. J. (1992). Physiology of thalamus and cortex. In A. N. Popper \& R. R. Fay (Eds.), The mammalian auditory pathway: Neurophysiology (vol. 2, pp. 232-334). New York: Springer-Verlag.

Delgutte, B., Joris, P. X., Litovsky, R. Y., \& Yin, T. C. (1999). Receptive fields and binaural interactions for virtual-space stimuli in the cat inferior colliculus. Journal of Neurophysiology, 81, 2833-2851.

Ehret, G., \& Merzenich, M. M. (1988). Neuronal discharge rate is unsuitable for encoding sound intensity at the inferior colliculus level. Hearing Research, 35, 1-8.

Fitzpatrick, D. C., Batra, R., Stanford, T. R., \& Kuwada, S. (1997). A neuronal population code for sound localization. Nature, 388, 871-874.

Fuzessery, Z. M., Wenstrup, J. J., \& Pollak, G. D. (1990). Determinants of horizontal sound location selectivity of binaurally excited neurons in an isofrequency region of the mustache bat inferior colliculus. Journal of

Neurophysiology, 63, 1128-1147.

Gaese, B. H., \& Johnen, A. (2000). Coding for auditory space in the superior colliculus of the rat. European Journal of Neuroscience, 12, 1739-1752.

Groh, J. M. (2001). Converting neural signals from place codes to rate codes. Biological Cybernetics, 85, 159-165.

Groh, J. M., \& Underhill, A. M. (2001). Coding of sound location in primate inferior colliculus. Society for Neuroscience Abstracts, 27, 60.1.

Groh, J. M., \& Sparks, D. L. (1992). Two models for transforming auditory signals from head-centered to eye-centered coordinates. Biology and Cybernetics, 67, 291-302.

Groh, J. M., Trause, A. S., Underhill, A. M., Clark, K. R., \& Inati, S. (2001). Eye position influences auditory responses in primate inferior colliculus. Neuron, 29, 509-518.

Harrison, J. M., \& Downey, P. (1970). Intensity changes at the ear as a function of the azimuth of a tone source: A comparative study. Journal of the Acoustical Society of America, 47, 1509-1518.

Hartung, K., \& Sterbing, S. (2001). A computational model of sound localization based on neurophysiological data. In S. Greenberg \& M. Slaney (Eds.), Computational models of auditory function (pp. 101-114). Amsterdam: IOS Press.

Hind, J. E., Goldberg, J. M., Greenwood, D. D., \& Rose, J. E. (1963). Some discharge characteristics of single neurons in the inferior colliculus of the cat: II. timing of the discharges and observations on binaural stimulation. Journal of Neurophysiology, 26, 321-341.

Ingham, N. J., Hart, H. C., \& McAlpine, D. (2001). Spatial receptive fields of inferior colliculus neurons to auditory apparent motion in free field. Journal of Neurophysiology, $85,23-33$.

Irvine, D. R. (1987). A comparison of two methods for the measurement of neural sensitivity to interaural intensity differences. Hearing Research, 30, 169-179.

Irvine, D. R., \& Gago, G. (1990). Binaural interaction in highfrequency neurons in inferior colliculus of the cat: Effects of variations in sound pressure level on sensitivity to interaural intensity differences. Journal of Neurophysiology, 63, 570-591.

Irvine, D. R. F. (1992). Physiology of the auditory brainstem. In A. N. Popper \& R. R. Fay (Eds.), The mammalian auditory pathway: Neurophysiology (vol. 2, pp. 153-231). New York: Springer-Verlag.

Itaya, S. K., \& Van Hoesen, G. W. (1982). Retinal innervation of the inferior colliculus in rat and monkey. Brain Research, 233, 45-52.

Jay, M. F., \& Sparks, D. L. (1987). Sensorimotor integration in the primate superior colliculus: II. Coordinates of auditory signals. Journal of Neurophysiology, 57, 35-55.

Jenkins, W. M., \& Masterton, R. B. (1982). Sound localization: Effects of unilateral lesions in central auditory system. Journal of Neurophysiology, 47, 987-1016.

Judge, S. J., Richmond, B. J., \& Chu, F. C. (1980). Implantation of magnetic search coils for measurement of eye position: An improved method. Vision Research, 20, 535-538.

Kelly, J. B., \& Kavanagh, G. L. (1994). Sound localization after unilateral lesions of inferior colliculus in the ferret (Mustela putorius). Journal of Neurophysiology, 71, 1078-1087.

Kelly, K. A., Metzger, R. R., Mullette-Gillman, O. A., Werner-Reiss, U., \& Groh, J. M. (2002). Representation of sound location in the primate brain. In A. Ghazanfar (Ed.), Primate audition: behavior and neurobiology (pp. 177-197). Boca Raton, FL: CRC Press.

King, A. J., \& Hutchings, M. E. (1987). Spatial response properties of acoustically responsive neurons in the superior colliculus of the ferret: A map of auditory space. Journal of Neurophysiology, 57, 596-624.

King, A. J., \& Palmer, A. R. (1983). Cells responsive to free-field auditory stimuli in guinea-pig superior colliculus: Distribution and response properties. Journal of Physiology, 342, 361-381.

Knudsen, E. I., \& Konishi, M. (1978). A neural map of auditory space in the owl. Science, 200, 795-797.

Kuwada, S., \& Yin, T. C. (1983). Binaural interaction in low-frequency neurons in inferior colliculus of the cat. I. Effects of long interaural delays, intensity, and repetition rate on interaural delay function. Journal of Neurophysiology, 50, 981-999.

Mascetti, G. G., \& Strozzi, L. (1988). Visual cells in the inferior colliculus of the cat. Brain Research, 442, 387-390.

Masterton, R. B. (1992). Role of the central auditory system in hearing: The new direction. Trends in Neuroscience, 15 , 280-285. 
Masterton, R. B., \& Imig, T. J. (1984). Neural mechanisms for sound localization. Annual Review of Physiology, 46, 275-287.

McAlpine, D., Jiang, D., \& Palmer, A. R. (1996). Interaural delay sensitivity and the classification of low best-frequency binaural responses in the inferior colliculus of the guinea pig. Hearing Research, 97, 136-152.

McAlpine, D., Jiang, D., \& Palmer, A. R. (2001). A neural code for low-frequency sound localization in mammals. Nature Neuroscience, 4, 396-401.

Meredith, M. A., \& Stein, B. E. (1986). Spatial factors determine the activity of multisensory neurons in cat superior colliculus. Brain Research, 365, 350-354.

Metzger, R. R., Mullette-Gillman, O. A., Underhill, A. M., Cohen, Y. E., \& Groh, J. M. (2003). Effect of initial eye position on saccades to auditory targets in monkeys. Society for Neuroscience Abstracts, in press.

Middlebrooks, J. C., \& Knudsen, E. I. (1984). A neural code for auditory space in the cat's superior colliculus. Journal of Neuroscience, 4, 2621-2634

Middlebrooks, J. C., Xu, L., Eddins, A. C., \& Green, D. M. (1998). Codes for sound-source location in nontonotopic auditory cortex. Journal of Neurophysiology, 80, 863-881.

Moore, D. R., Hutchings, M. E., Addison, P. D., Semple, M. N., \& Aitkin, L. M. (1984). Properties of spatial receptive fields in the central nucleus of the cat inferior colliculus: II. Stimulus intensity effects. Hearing Research, 13, 175-188.

Moore, D. R., Semple, M. N., Addison, P. D., \& Aitkin, L. M. (1984). Properties of spatial receptive fields in the central nucleus of the cat inferior colliculus: I. Responses to tones of low intensity. Hearing Research, 13, 159-174.

Musicant, A. D., Chan, J. C., \& Hind, J. E. (1990). Direction-dependent spectral properties of cat external ear: New data and cross-species comparisons. Journal of the Acoustical Society of America, 87, 757-781.

Palmer, A. R., Rees, A., \& Caird, D. (1990). Interaural delay sensitivity to tones and broad band signals in the guinea-pig inferior colliculus. Hearing Research, 50, 71-86.

Phillips, D. P., \& Brugge, J. F. (1985). Progress in neurophysiology of sound localization. Annual Review of Psychology, 36, 245-274.

Ramachandran, R., Davis, K. A., \& May, B. J. (1999). Single-unit responses in the inferior colliculus of decerebrate cats: I. Classification based on frequency response maps. Journal of Neurophysiology, 82, 152-163.

Robinson, D. (1963). A method of measuring eye movement using a scleral search coil in a magnetic field. IEEE Transactions of Biomedical Engineers, 10, 137-145.

Rose, J. E., Greenwood, D., Goldberg, J., \& Hind, J. (1963). Some discharge characteristics of single neurons in the inferior colliculus of the cat: I. Tonotopical organization, relation of spike counts to tone intensity, and firing patterns of single elements. Journal of Neurophysiology, 26, 294-320.

Ryan, A., \& Miller, J. (1977). Effects of behavioral performance on single-unit firing patterns in inferior colliculus of the rhesus monkey. Journal of Neurophysiology, 40, 943-956.

Ryan, A., \& Miller, J. (1978). Single unit responses in the inferior colliculus of the awake and performing rhesus monkey. Experimental Brain Research, 32, 389-407.

Semple, M. N., Aitkin, L. M., Calford, M. B., Pettigrew, J. D., \& Phillips, D. P. (1983). Spatial receptive fields in the cat inferior colliculus. Hearing Research, 10, 203-215.

Semple, M. N., \& Kitzes, L. M. (1987). Binaural processing of sound pressure level in the inferior colliculus. Journal of Neurophysiology, 57, 1130-1147.

Spezio, M. L., Keller, C. H., Marrocco, R. T., \& Takahashi, T. T. (2000). Head-related transfer functions of the Rhesus monkey. Hearing Research, 144, 73-88.

Stanford, T. R., Kuwada, S., \& Batra, R. (1992). A comparison of the interaural time sensitivity of neurons in the inferior colliculus and thalamus of the unanesthetized rabbit. Journal of Neuroscience, 12, 3200-3216.

Sterbing, S. J., Hartung, K., \& Hoffmann, K. P. (2002). Representation of sound source direction in the superior colliculus of the guinea pig in a virtual auditory environment. Experimental Brain Research, 142, 570-577.

Stillman, R. D. (1972). Responses of high-frequency inferior colliculus neurons to interaural intensity differences. Experimental Neurology, 36, 118-126.

Takahashi, T. T. (1989). The neural coding of auditory space. Journal of Experimental Biology, 146, 307-322.

Wagner, H. (1993). Sound-localization deficits induced by lesions in the barn owl's auditory space map. Journal of Neuroscience, 13, 371-386.

Wise, L. Z., \& Irvine, D. R. (1983). Auditory response properties of neurons in deep layers of cat superior colliculus. Journal of Neurophysiology, 49, 674-685.

Wise, L. Z., \& Irvine, D. R. (1985). Topographic organization of interaural intensity difference sensitivity in deep layers of cat superior colliculus: Implications for auditory spatial representation. Journal of Neurophysiology, 54, 185-211.

Yin, T. C., Chan, J. C., \& Irvine, D. R. (1986). Effects of interaural time delays of noise stimuli on low-frequency cells in the cat's inferior colliculus: I. Responses to wideband noise. Journal of Neurophysiology, 55, 280-300.

Yin, T. C., Kuwada, S., \& Sujaku, Y. (1984). Interaural time sensitivity of high-frequency neurons in the inferior colliculus. Journal of the Acoustical Society of America, 76, 1401-1410.

Zrull, M. C., \& Coleman, J. R. (1997). Effects of tectal grafts on sound localization deficits induced by inferior colliculus lesions in hooded rats. Experimental Neurology, 145, $16-23$. 